\title{
LA INFLUENCIA DE LAS SERIES DE MÉDICOS EN LOS ESPECTADORES Y EN LOS PROFESIONALES DE LA SALUD
}

\section{THE INFLUENCE OF MEDICAL TV SERIES IN VIEWERS AND HEALTH PROFESSIONALS}

Graciela Padilla Castillo1: Universidad Complutense de Madrid (España). gracielapadilla@ccinf.ucm.es

\section{RESUMEN}

Los manuales de comunicación y de ética sanitaria han quedado relegados a las facultades de Medicina y de Enfermería. Esta investigación pretende romper esa tradición y llevar esos estudios a las facultades de comunicación, donde contamos con herramientas y metodologías de investigación realmente valiosas. Por ejemplo, las series de televisión de médicos suponen un amplio terreno de estudio. Estudiaremos las series norteamericanas de médicos, estrenadas en España, hasta 2010. Los guionistas toman su inspiración de la prensa, de la realidad, de los asuntos que interesan a la sociedad. Por ello, ofrecen importantes retratos del tratamiento del sida, el cáncer, el aborto, el Parkinson, el Alzheimer, el consentimiento informado, la protección de datos, la intimidad del paciente, el trasplante de órganos o la comunicación de malas noticias. Para el análisis cualitativo, nos hemos valido de las teorías de Ole R. Holsti; las Estrategias de Comunicación que identificó Ray Eldon Hiebert; la definición de Ética, Moral y Política de Gustavo Bueno y la clasificación de las relaciones de dependencia que distinguió Sánchez Corredera. Los resultados revelan que este tipo de ficción está tan pegado a la realidad que influye en el espectador como posible paciente 0 familiar del paciente.

PALABRAS CLAVE: Ética - comunicación sanitaria - ficción televisiva - series de médicos.

\section{ABSTRACT}

Communication and health manuals about ethics have been relegated to the Faculties of Medicine and Nursing. This proposal aims to break this tradition and bring these studies to the powers of communication, where we have research tools and research methodologies really valuable. For example, medical TV series include a wide field of study. In this paper, we study the North American medical series, released in Spain until 2010. Its writers take their inspiration from the press, the reality and the issues that concern to society. Therefore provide important portraits

\footnotetext{
${ }^{1}$ Graciela Padilla Castillo: Doctora en Ciencias de la Información (2010) por la Universidad Complutense de Madrid, con Premio Extraordinario de Doctorado, y Licenciada en Periodismo y Comunicación Audiovisual (2006), con Premio Fin de Carrera. Ha completado su formación posdoctoral en la Universidad de California en Los Ángeles (UCLA). Es profesora de la asignatura "Ética y Deontología profesional” en los Grados y Licenciaturas de Periodismo, Comunicación Audiovisual, y Publicidad y Relaciones Públicas.
} 
treatment of AIDS, cancer, abortion, Parkinson's, Alzheimer's, informed consent, data protection, patient privacy, organ transplantation or communication of bad news. For qualitative analysis, we have used the theories of Ole Holsti; Communication Strategies identified by Ray Eldon Hiebert; the definition of Ethics, Moral and Political by Gustavo Bueno; and classification of the dependency relationships that distinguished Sánchez Corredera. The results show that this kind of fiction is so close to reality that influences the spectator as possible patient or patient's family.

KEY WORDS: Ethics - health communication - fiction television - TV series of doctors.

Graciela Padilla Castillo. (2012). LA INFLUENCIA DE LAS SERIES DE MÉDICOS EN LOS ESPECTADORES Y EN LOS PROFESIONALES DE LA SALUD. Revista de Ciencias de la Comunicación e Información. (Junio 2012). Año XXVI (29), 21-33 ISSN: 0213-070-X. Recuperado: http://www.revistaccinformacion.net/ARTICULOS/2012\%20e-

\%20Ang\%C3\%A9lica/2012.\%20La\%20influencia\%20de\%20las\%20series\%20de\%20m\% C3\%A9dicos.\%20Graciela\%20Padilla.pdf

\section{INTRODUCCIÓN:}

Esta investigación analiza la comunicación en las series de televisión de médicos, que trabajan en hospitales, de 1990 a 2010. Dentro de ese formato, hemos elegido todas las series de producción norteamericana, estrenadas en España. El elevado número de temporadas de esas series nos ha obligado a establecer un marco espacio-temporal concreto. Por ello, estudiamos únicamente la primera temporada de cada serie. Este lapso de emisiones incluye el capítulo piloto, que sienta las bases, ideas y características fundamentales de los personajes; y los primeros capítulos, que siguen fielmente las ideas originales de cada proyecto. El éxito o fracaso de las series puede obligar a cambiar el tono, los temas e incluso, los personajes originales.

Queremos remarcar que son todas series ambientadas en hospitales, donde hay sanitarios y pacientes. Las series que se desarrollaban sólo en una consulta privada, ajena a un hospital o clínica, han sido desestimadas porque apenas contienen información y comunicación sanitaria.

\section{OBJETIVOS:}

El propósito principal es examinar y comparar las series elegidas para identificar las modalidades de comunicación que hay en ellas. A partir del estudio de los protagonistas, tramas y diálogos, pretendemos descifrar su éxito y entender la posible empatía e identificación entre personajes de ficción y espectadores. Los protagonistas se convierten en amigos, confidentes y casi familiares del público, que conecta con ellos emocionalmente. Dentro de un análisis más profundo, pretendemos aplicar ese análisis a unos campos concretos:

- Comunicación institucional (sanitario-paciente, sanitario-familiares del paciente y sanitario-sanitario), en la que consideramos la tipología de acontecimientos públicos y las estrategias de comunicación que emplean 
los profesionales de la salud para solucionar los problemas internos y externos de los hospitales.

- Relaciones entre la Ética, la Moral y la Política, dentro de los hospitales y entre los sanitarios.

Estos campos de la comunicación dan valor e importancia a los resultados de esta investigación. La hipótesis es que las series se alimentan de la realidad y devuelven esa inspiración, influyendo sobre ella. Esta retroalimentación cíclica se da desde el comienzo de las series de médicos, pero es más palpable en el periodo estudiado porque existen estudios, entrevistas y datos sobre la satisfacción del usuario de la Sanidad, los motivos que llevan a los jóvenes a estudiar Medicina, o los términos médicos y enfermedades que los ciudadanos buscan en Internet tras escucharlos en la pequeña pantalla. Las series que tratamos en las páginas siguientes son, por lo tanto, mucho más que ficción.

Además, la elección del tema de investigación responde al éxito prolongado de las series de médicos en la ficción televisiva. En 1956, se estrenó en España la primera de ellas, Dr. Christian. Era de origen norteamericano y se mantuvo una sola temporada, con 15 episodios. Desde entonces, los guionistas han creado decenas de seriales médicos, en tono de drama y de humor, y en muchos países del mundo. Los índices de audiencia son muy altos. Algunas series, como Anatomía de Grey, Sin cita previa, House, Nip/Tuck, a golpe de bisturí o Nurse Jackie se han convertido en las más vistas en su país de origen, Estados Unidos. Su triunfo se repite al mismo tiempo en otros países, como España, que adquieren los derechos de emisión. Todo lo anterior justifica la relevancia de este estudio. Pretendemos comprender el éxito de este tipo de ficción y abrir una nueva vía de conocimiento y de investigación en comunicación y ética sanitarias.

\section{HIPÓTESIS:}

El objeto de estudio pretende confirmar las siguientes hipótesis, surgidas tras el primer visionado de las series:

- Estas series nos muestran formas reales de comunicación interpersonal en tres variables: sanitario-paciente, sanitario-familiares del paciente y sanitariosanitario. El sintagma "sanitario" cubrirá, en adelante, a médicos/as, enfermeros/as y celadores/as. Esas formas de comunicación nos interesan porque los públicos pueden elegir aquellas series en las que aparezcan las mejores pautas de comunicación institucional en acontecimientos rutinarios.

- Las maneras que los profesionales de los hospitales tienen de solucionar los accidentes, escándalos y acontecimientos fortuitos pueden ser factores que también influyan en las elecciones de las series.

- Los accidentes, escándalos y acontecimientos fortuitos, al basarse en hechos reales, que según el año y la época en que se estrenó cada serie van variando, pueden servir de modelos de comportamiento de solucionar los problemas a los profesionales de la salud; de ahí que aquí puede radicar la continuidad de un público específico fiel, que puede contribuir al éxito de las series.

- Cada episodio plantea una relación de dependencia entre Ética, Moral y 
Política. De ahí que, si predominan decisivamente unas relaciones sobre otras, podemos considerar que ésas son las relaciones que los públicos quieren que predominen en el sistema de salud $y$, por eso, siguen asiduamente esas series.

\section{METODOLOGÍA:}

Para este trabajo de investigación, hemos utilizado un procedimiento analíticosintético. Centramos el primer paso exploratorio en buscar las series de médicos de mayor éxito desde 1990 hasta 2010. El segundo paso incluye el análisis de todos los episodios de la primera temporada de las series elegidas. Por último y en tercer lugar, interpretamos esos capítulos con un análisis de contenido cuantitativo y cualitativo, interpretando los resultados. Para ello, nos hemos valido del libro de Ole R. Holsti que, a nuestro entender, todavía no ha sido superado. Al mismo tiempo, examinamos fuentes primarias especializadas (libros, artículos y noticias). Completamos ese análisis de contenido con: la teoría sobre acontecimientos públicos de Harvey Molotch y Marilyn Lester; las Estrategias de Comunicación que identificó Ray Eldon Hiebert y, que también a nuestro entender, todavía no han sido superadas; la definición de Ética, Moral y Política de Gustavo Bueno y la clasificación de las relaciones de dependencia entre las citadas E-M-P, que distinguió Silverio Sánchez Corredera.

Pretendemos cubrir dos décadas completas de emisiones. Las series que entran en este marco son las que especificamos a continuación. El primer intervalo corresponde a su emisión en Estados Unidos y la fecha concreta entre paréntesis se refiere a su estreno en España:

- Doctor en Alaska: 1990-1995 (12 de julio de 1990).

- La Doctora Quinn: 1993-1998 (1 de enero de 1993).

- Chicago Hope: 1994-2000 (18 de septiembre de 1994).

- Urgencias: 1994-2009 (19 de septiembre de 1994).

- Becker: 1998-2004 (2 de noviembre de 1998).

- Doctoras de Filadelfia: 2000-2006 (23 de julio de 2000).

- Doc: 2001-2004 (11 de marzo de 2001).

- Scrubs: 2001-2010 (2 de octubre de 2001).

- Nip/Tuck. A golpe de bisturí: 2003-2010 (22 de julio de 2003).

- House: 2004-2010 (16 de noviembre de 2004).

- Anatomía de Grey: 2005-2010 (27 de marzo de 2005).

- Saved: 2006 (12 de junio de 2006).

- 3 libras: 2006 (14 de noviembre de 2006).

- Sin cita previa: 2007-2010 (19 de septiembre de 2007).

- Mental: 2009 (4 de junio de 2009).

- Nurse Jackie: 2009-2010 (8 de abril de 2010).

- Mercy: 2009-2010 (25 de noviembre de 2009).

- Trauma: 2009-2010 (24 de noviembre de 2009).

- ThreeRivers: 2009 (8 de abril de 2010).

Del mismo modo, podemos recomendar algunas lecturas académicas que también han analizado la ética y la comunicación en ficción televisiva y cinematográfica y que 
han ayudado en el marco metodológico de esta investigación: Aladro (2011), Padilla (2009, 2010a y 2010b), Requeijo (2010, 2012a y 2012b), Rodríguez Vidales (2012) y Valbuena (2006a, 2006b, 2007a, 2007b, 2009 y 2010).

\section{MARCO TEÓRICO:}

La Definición es uno de los cuatro "modisciendi" o "rasgos de segundo orden" que tiene una Ciencia. Los otros tres son: Divisiones/Clasificaciones, Modelos y Demostraciones (Bueno, 1987: 335; Valbuena, 1997: 61-64). Desde hace tiempo, venimos observando el gran descuido con que algunos "teóricos" tratan las definiciones de sus disciplinas. Y si esto es así, las bases de cualquier disciplina se tambalean.

Siguiendo a Gustavo Bueno, podemos dar una definición efectiva e intencional. El campo efectivo de cualquier disciplina, tal como aparece en los libros, investigaciones y estudios realizados hasta ahora, es in-fecto, no acabado: no todas las relaciones entre los términos que enuncian los investigadores y teóricos pueden verse acompañadas por las operaciones que deben entretejerse con estas relaciones. Además, hay teorías particulares que no cumplen, o cumplen a duras penas, los otros trámites o momentos semánticos y pragmáticos. Nosotros podemos adaptar esas definiciones a la comunicación institucional de la siguiente manera:

- Partiendo de una organización realmente existente, como un Hospital, y particularmente, si esta organización está conformada por profesionales con diversas funciones y por pacientes, que reciben los servicios de un sistema de salud;

- Entendemos por comunicación institucional del hospital como la que se establece entre sanitario-pacientes, sanitario-familiares del paciente y sanitario-sanitario;

- Teniendo en cuenta que este tipo de comunicación puede desarrollarse en un ambiente tranquilo, reactivo, turbulento o autorrecreado, por lo que quienes conforman el sistema de salud pueden estar inmersos en actividades rutinarias, accidentes, escándalos o acontecimientos fortuitos, que exigen respuestas que solucionen los problemas.

El campo intencional o ideal de cualquier disciplina es el que alcanzará su perfección cuando las operaciones saturen las proposiciones científicas de las diversas teorías. El ideal interno de cualquier disciplina de la Información y de la Comunicación es lograr la conformidad o paralelismo entre sus relaciones y operaciones. En consecuencia, definimos intencionalmente la comunicación institucional del Hospital como aquella relación por la que sanitarios y pacientes:

- Informan, aumentando las probabilidades de elección de los pacientes entre los planes y programas que ofrecen; o desinforman, disminuyendo las probabilidades de elección entre los planes y programas de los profesionales de la salud o de los pacientes.

- Motivan, cambiando los valores relativos que sanitarios y pacientes como receptores atribuyen a los posibles resultados de su acción para impulsar sus 
planes y programas o disuaden a los receptores para que no acepten determinados planes y programas.

- Instruyen, indicando las eficiencias de cualquiera de las vías de acción necesarias para implantar los planes y programas, o señalando las insuficiencias de vías alternativas.

- Todo ello para lograr el buen orden social o eutaxia del Hospital.

Para mostrar la evolución y desarrollo de la Comunicación Institucional, tendremos en cuenta las dos definiciones que hemos ofrecido. Un buen punto de partida es el que ofrece Laín Entralgo (2003: 41), quien afirmaba que esta ciencia entra en la vida del hombre cuando hay dolor. Nuestro cuerpo nos muestra su importancia cuando duele y sólo entonces. Ahí es cuando el hombre pide ayuda al sanitario y tiene lugar el acto médico: un encuentro entre dos seres humanos, "determinado en su contenido, en su figura y en su curso por la intención consciente e inconsciente de uno y otro en el momento de encontrarse" (Laín, 2003: 42). La intención del médico es la voluntad de ayuda y la intención del paciente es la voluntad de curación.

La época actual, en la que se desarrollan las series de televisión de esta investigación, es la era de la comunicación y paradójicamente, falta una concepción clara y distinta de la comunicación institucional. Aunque nos resulta muy insatisfactoria, presentamos la definición de salud de la Organización Mundial de la Salud (OMS), como "estado de bienestar completo físico, psíquico y social y no solamente la ausencia de enfermedad o invalidez. Se rompe, así, con el reduccionismo fisiológico de salud vs. enfermedad y se la inserta en una dimensión integral humana en el sentido bio-psico-social" (Chimeno, en Orive, 1994: 60).

Elegimos esta definición porque sólo el diálogo y la comunicación con el paciente van más allá de la fisiología. El médico no es el único responsable de la curación y el paciente debe participar en el proceso. Ese diálogo, esa participación y esa comunicación facilitan la experiencia individual de ir al médico. Y el sanitario debe manejar estas variables para informar a su paciente:

- Autonomía del paciente.

- Llamar al paciente por su nombre.

- Derecho a la información del paciente.

- Derecho a la intimidad del paciente.

- Derecho a la confidencialidad de los datos médicos.

- Escucha activa y empatía.

- Los signos de la enfermedad.

- Escucha activa y presencia del paciente.

- El nivel de implicación.

\section{RESULTADOS SOBRE ACONTECIMIENTOS PÚBLICOS Y CRISIS:}

Dentro de la Comunicación Institucional, son tan variados los hechos que es necesario disponer de un armazón teórico sólido para afrontarlos. Contamos con ese armazón, que es el de la Tipología de los Acontecimientos Públicos, de Harvey Molotch y Marilyn Lester. El diagrama principal de su teoría aparece a continuación: 


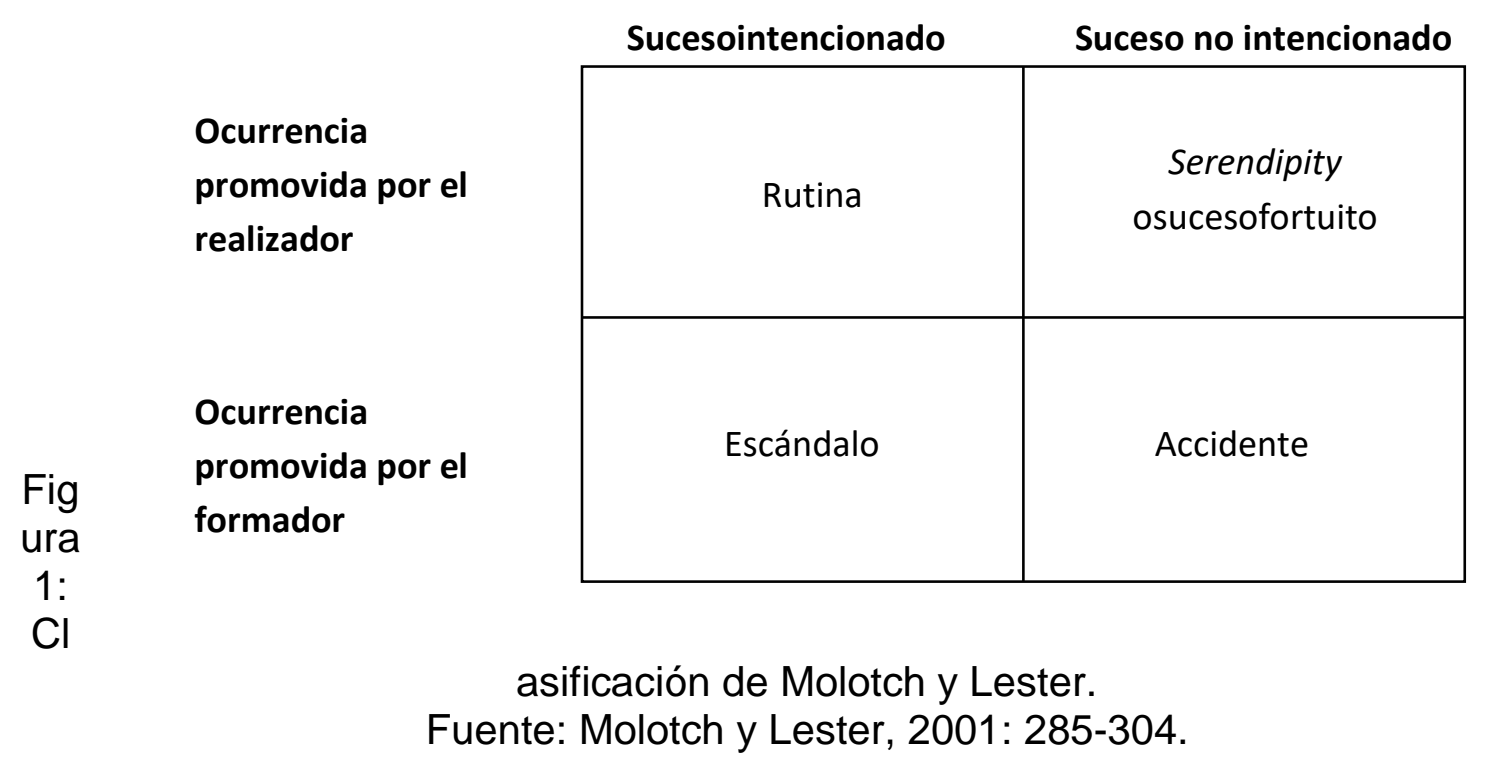

Nos centraremos sólo en los escándalos y en los accidentes. En todas las series sobre médicos hay, al menos, una crisis. Los episodios de La doctora Quinn comenzaron incluyendo una crisis en cada uno. ThreeRivers, la máxima evolución, desarrolla tres crisis por capítulos: tres fallecimientos, tres donantes, tres receptores, seis familias. Así se distribuyen esas crisis por temas en todas las series estudiadas:

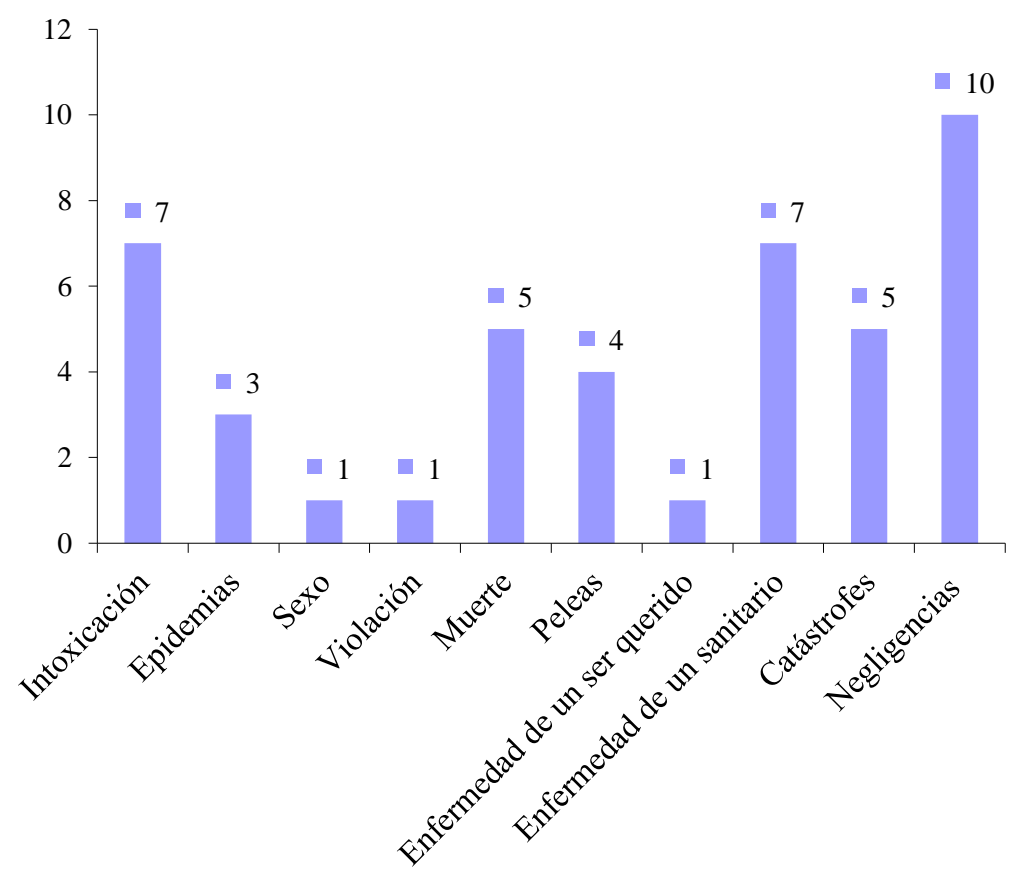

Figura 2: Temáticas de las crisis de las series de médicos.

Fuente: Elaboración propia. 
Trasladando esa abundancia de crisis a la realidad, sabemos que la dirección de comunicación de un Hospital ha de contar con su propio anexo al plan de emergencias de la empresa o institución en que trabaja. Este anexo ha de describir:

- Antecedentes de crisis que hayan existido en organizaciones de la misma actividad.

- Casos simulados de crisis.

- Quiénes forman el comité de crisis y si es necesario contar con asesoría externa.

- Qué canales utilizará para transmitir información (repertorio de contactos personales antes diversas instancias, fichero de periodistas especializados).

- Qué responsables enviar al escenario de los hechos según el tipo de emergencia de que se trate.

- Qué relación ha de establecerse con el alto cargo oficial de la institución en el escenario de los hechos.

Asimismo, ha de contener la información completa de los altos mandos de la empresa o institución. Incluso cuando se cuenta con este tipo de planes y todo está bien planteado, pueden existir desacuerdos intensos o solapamientos a la hora de actuar. Durante las emergencias, y al margen de estos planes de actuación, la experiencia de los altos cargos de las instituciones y del responsable de comunicación son el factor decisivo para el éxito o el fracaso en la comunicación con el público. De todos modos, es posible establecer líneas específicas de actuación en materia de comunicación en los casos de emergencia. Estas líneas pueden ser útiles para convertir un desastre público en un éxito de la dirección de comunicación. Y las series de televisión, con sus ejemplos, proporcionan algunas directrices sobre comunicación sanitaria:

- Decidir si el portavoz va a ser un responsable ejecutivo de la empresa o institución o el responsable de comunicación.

- Centralizar la función de informar. Si el responsable de la empresa o institución no centraliza, encontrará confusiones sobre la exactitud de los comentarios y sobre la autoridad y responsabilidad de quienes los emite.

- Dejar claro, desde el principio, que el responsable de la dirección de comunicación o del gabinete de prensa es el personaje al que los medios pueden acudir para obtener información o trabajar sobre ella.

- El responsable de comunicación tiene que informar directamente al responsable de la empresa o institución de quien dependa; a su vez, tiene que participar en sus decisiones, y acceder a toda la información, sea confidencial o de otro tipo.

- Cuando no sea posible verificar la información, citar a alguna autoridad que la suscriba, en lugar de darla como de propia cosecha.

- Responder rápidamente, y con autoridad, a las preguntas de los medios, pero no apresurarse en las respuestas sin comprobar primero un pleno conocimiento de las mismas.

- Hablar claramente. Cuando la información puede provocar el pánico o perjuicio a personas inocentes, seleccionar muy cuidadosamente qué decir y qué callar.

- Presentar hechos; no opiniones. Dejar que los medios saquen sus propias conclusiones sobre las actuaciones de la empresa o institución. 
- Recordar que los medios tienen una misión distinta de la dirección de comunicación o del gabinete de prensa. Ellos deben recoger y difundir noticias; el gabinete, informar sobre las actuaciones, programas o servicios.

- Cuando no se disponga de hechos, ayudar a los medios a conseguir material de apoyo. Los periodistas que cubren crisis necesitan llenar sus archivos. La falta de información siempre da como resultado que surjan mensajes "creativos" basados en la desinformación, la suposición o el rumor.

- Independientemente de las consecuencias, el responsable de comunicación ha de incluirse a sí mismo en la toma de decisiones del programa, si existe un interés desacostumbrado del público por ser informado. Su conciencia ha de dictar, en último término, sus actuaciones.

- Usar todos los medios disponibles para informar al público afectado. El coste monetario de estos medios es un factor secundario en las situaciones de emergencia, aun incurriendo en gastos no presupuestados.

- Mantener el sentido del humor, pero no mostrarlo. El humor puede ser mal interpretado en una crisis y, después de todo, cada comunidad tiene sus propios comediantes.

- Cuando la emergencia acabe, hay que dejar que el flujo de información vuelva a su cauce. Si el gobierno se inmiscuye durante largos períodos en los canales de comunicación establecidos, pueden producirse daños muy grandes.

- Disfrutar de la satisfacción que supone comunicar información que salva vidas y propiedades. Cuando la emergencia termine, todos recogerán los frutos o los perjuicios de las actividades del programa, pero cuando una estrategia de comunicación consigue el éxito, pocas personas se dan cuenta.

\section{RESULTADOS SOBRE ÉTICA, POLÍTICA Y MORAL SANITARIA:}

Hemos escogido este enfoque porque creemos que uno de los factores que explica el éxito de estas series es comprobar cómo sus protagonistas, trabajando muchas veces en un ambiente turbulento por la gran cantidad de estímulos y desafíos a que tienen que hacer frente, saben mantener unos valores consistentes y beneficiosos para la sociedad. De manera que, si comparamos estas series con otras, comprobamos que los valores son diferentes; incluso, muy diferentes.

Ética es todo aquello que afecta a las personas en cuanto son individualizables (no aisladas), en cuanto a sujetos distributivos dentro de un grupo. Es toda praxis humana que contribuya a mantener la fortaleza del sujeto humano y, por tanto, todo comportamiento que suponga el respeto por la integridad del ser humano corpóreo. La fortaleza se entenderá como firmeza, cuando vaya referida a uno mismo, y como generosidad, cuando vaya dirigida a los demás. Conductas antiéticas son las que socavan la fortaleza de los otros produciendo daños a su imagen pública, a su hacienda 0 , directamente, a su integridad corporal (malos tratos, lesiones, homicidios, etc.) (Alvargonzález, 2009: 21). La desatención hacia el propio cuerpo, el descuido relativo a nuestra salud, es también un delito ético, por lo que tiene de falta de firmeza. La medicina es una actividad que marcha paralelamente al curso de las virtudes éticas. Podría decirse que la ética es a la medicina lo que la moral es a la política (Bueno, en García Sierra, 2000: 473-474). 
Moral es todo lo que afecta a los individuos o grupos sociales en cuanto los consideramos atributivamente como partes de la sociedad sin posibilidad de operar en términos simétricos unos con otros. El principio fundamental de la moralidad es la justicia, entendida como la aplicación escrupulosa de las normas que regulan las relaciones de los individuos o grupos de individuos en cuanto partes del todo social: "La fuerza de obligar (o impulso) de las normas morales procede, no tanto del individuo, cuanto del control o presión social del grupo, canalizado a través de un código deontológico o de un sistema de "leyes no escritas" y, no por ello, menos coactivas" (Bueno, en García Sierra, 2000: 477-478).

Política es algo en principio próximo a la moral, por cuanto considera también a los individuos o grupos sociales atributivamente, pero esta vez no en torno a la idea de justicia sino en torno al simple "buen orden social": "Lo esencial es tener en cuenta que el poder político implica siempre la inserción del poder en el contexto de programas y planes orientados a la eutaxia - «buen orden social» - de una sociedad dada, y ésta es la razón por la cual suponemos que el poder político es indisociable de la palabra, como instrumento suyo" (Bueno, en García Sierra, 2000: 563-564).

Silverio Sánchez Corredera es discípulo de Gustavo Bueno y ha prolongado la triple distinción, enfocándola desde la perspectiva de las relaciones. Dedicó la primera parte de su Tesis Doctoral a este asunto y, a nuestro parecer, abrió una vía original de investigación en Comunicación. Las relaciones entre $E$ (ética), $M$ (moral) y $P$ (política) son dialécticas y tantas cuantos casos entreverados puedan registrarse en los hechos. Sin embargo, podemos diseñar algunas figuras básicas características. Estos tres campos registran fenómenos de no relación, de contradicción, de complementariedad, de dependencia, etc. Vamos a fijarnos, en particular, en las relaciones de dependencia de unas respecto de las otras (Sánchez Corredera, 2003: 40). Este autor ofrece 12 relaciones de dependencia (Sánchez Corredera, 2003: 3945) y esas relaciones nos permiten estudiar una organización con tantas variables como un Hospital.

Así, este apartado presenta otro análisis de contenido cuantitativo y cualitativo. Cuantitativamente, tomamos como Categoría la relación E-M-P; como unidad de registro, el episodio; como unidad de contexto, la primera temporada. Cualitativamente, analizamos cada episodio y las relaciones que aparecen en él, identificando las relaciones de la 1) a la 12). Tras contabilizar todos, en los capítulos de las 19 series, sumamos un total de 387 casos, que bien nos pueden dar la imagen de las relaciones E-M-P. El amplio marco elegido hace que los resultados sean genuinos, originales y sobre todo, representativos de cómo son los sanitarios de la ficción, al menos, entre 1990 y 2010. Dentro de cada relación estudiada, se distribuyen de la siguiente manera:

\begin{tabular}{|l|l|}
\hline RELACIÓN & NÚMERO DE CASOS \\
\hline 1 & 110 \\
\hline 3 & 203 \\
\hline 6 & 2 \\
\hline 7 & 9 \\
\hline
\end{tabular}




\begin{tabular}{|l|l|}
\hline 8 & 7 \\
\hline 9 & 4 \\
\hline 10 & 6 \\
\hline 11 & 41 \\
\hline 12 & 5 \\
\hline TOTAL & 387 \\
\hline
\end{tabular}

Figura 3: Casos y relaciones E-M-P. Fuente: Elaboración propia.

Interpretamos estos resultados de la manera siguiente. Los seguidores de estas series admiran a los profesionales que sacrifican sus intereses individuales a los de las personas que conforman los grupos de los pacientes y de otros profesionales de la salud, es decir, prefieren la Relación 3 - Relaciones entre aspectos morales y éticos, de tal forma que los éticos se dan dependientemente de los morales-.

No resulta difícil interpretar la Relación 1 - Relaciones entre aspectos éticos y morales, de tal forma que los morales se dan dependientemente de los éticos-, que ocupa el segundo lugar, aunque los resultados pudieran resultar paradójicos. Por una parte, los guionistas quieren a pacientes que muestren su deseo de perdurar, de luchar por su salud, de no abandonar.

Ahora bien, esta relación puede ser el resultado de un proceso. La misma situación 1, puesta al comienzo de un episodio, puede resultar cacoética, es decir, que los médicos, paramédicos o pacientes otorguen más importancia a sus intereses individuales a corto plazo, que a plazo intermedio o largo.

También es lógico que la Relación 11 - Relaciones entre aspectos políticos, morales y éticos, de forma que los éticos son dependientes de los morales y ambos a su vez de los políticos - aparezca en tercer lugar, muy destacada de las restantes, aunque también representa un porcentaje más de un 50 inferior al de la situación 1. Un sistema de salud no puede subsistir si únicamente depende de factores psicológicos, comunicativos y organizativos. Porque el sistema de salud ha de tener una política. Y en no pocos casos, es perjudicial sobrepasar los límites de la política sanitaria. En ciertos casos, también, lo peor que le puede ocurrir a la comunicación sanitaria es estar subordinada a los intereses políticos, porque entonces se resiente la moral de la organización y la ética de los individuos.

\section{DISCUSIÓN:}

Después de haber explorado las personalidades y acciones de 89 personajes en 19 series de médicos de 1990 a 2010, los resultados configuran un sanitario ideal y favorito del público. Éste ejerce su trabajo de manera objetiva y valora éticamente todas las posibilidades para enfrentarse al mundo. Es responsable, eficaz, analítico, racional y excelente profesional. Antes de ser un personaje empático, cariñoso, amable, irónico o divertido, es un buen médico y un serio profesional. Convence a los espectadores en el desempeño de su trabajo. Además, es protector y paternalista. Ayuda y protege al paciente y a sus compañeros. Aporta confianza y 
porque se interesa por los demás. Los espectadores prefieren los médicos amables, que escuchan y apoyan.

Al lado de este sanitario, muchas veces hay otro (y que es el mismo) de carácter impetuoso, irracional y apasionado. Esta segunda elección permite la convivencia con sanitarios que se alejan de la típica imagen formal, seria y circunspecta de la profesión para resultar más cercanos al público. Pero sólo aparece en los contextos personales de los sanitarios; nunca en el hospital. El público no podría confiar en ellos al 100 por ciento.

Por nuestras conclusiones, también podemos aventurar que triunfarán siempre series corales, con personajes divididos entre lo profesional y lo personal, protectores y altruistas en el Hospital, y a la vez, adultos y éticos en la práctica de su trabajo. Existen desde 1956, con Dr. Christian, y rebosan en la parrilla actual con varias series simultáneas. Su supervivencia está asegurada si siguen siendo sanitarios humanos y verosímiles. Nada importa más al ser humano que su propia salud, y las series de médicos atienden esas inquietudes, reflejan el dolor y la alegría más sinceros, y hablan de los propios seres humanos. Su comportamiento, sus relaciones y sus sentimientos les hacen casi palpables, al otro lado de la pequeña pantalla. Todo ello da valor a la ficción televisiva como fuente de inspiración para la comunicación interpersonal e institucional. El espectador consume esta ficción y se informa y se forma como futuro usuario de la sanidad.

\section{REFERENCIAS:}

\section{Libros:}

Alvargonzález, D. (2009). La clonación, la anticoncepción y el aborto en la sociedad biotecnológica. Oviedo: Pentalfa.

Bueno, G. (1987). Etnología y utopía.Gijón: Pentalfa.

Bueno, G.(2000). Televisión:Apariencia y Verdad. Barcelona: Gedisa.

Laín, P. (2003). El médico y el enfermo. Madrid: Editorial Triacastela.

Orive, P. (ed.) (1994). Comunicación sanitaria. Madrid: Dossat 2000.

Valbuena, F. (1997). Teoría General de la Información. Madrid: Noesis.

Valbuena, F. (ed.) (2006 a). Eric Berne, teórico de la comunicación. Madrid: Edipo.

Artículos en revistas:

Aladro, E. (2011). La Teoría de la Información ante las nuevas tecnologías de la comunicación, en CIC Cuadernos de Información y Comunicación, nำ16, p. 8393.

Molotch, H. y Lester, M. (2001). Las noticias como conducta intencionada: sobre el 
uso estratégico de los acontecimientos rutinarios, los accidentes y los escándalos, en CIC Cuadernos de Información, no 6, junio, p. 285-304. Traducción de Aladro Eva.

Padilla Castillo, G. (2009). El éxito de Mujeres desesperadas desde el análisis transaccional, en Revista de Análisis Transaccional y Psicología Humanista, no 60, p. 20-35.

Padilla Castillo, G. (2010b). Los antihéroes televisivos desde las perspectivas del Análisis Transaccional, la Ética, la Moral y la Política, en Revista de Análisis Transaccional y Psicología Humanista, no 62, p. 81-101.

Requeijo, P. (2010). Mad Men desde el Análisis Transaccional, en Revista de Análisis Transaccional y Psicología Humanista, no 63, p. 261-279.

Requeijo Paula (2012b). Los juegos y los timos en Luna Nueva, Tener y no tener y Río Bravo, en Revista de Análisis Transaccional y Psicología Humanista, no 64, p. 106-127.

Sánchez Corredera, S. (2003). Los conflictos entre Ética, Moral y Política: criterios para su negociación, en CIC Cuadernos de Información, nº 8, junio, p. 39-60.

Valbuena, F.(2006b). Análisis de la película Esencia de mujer (1992), en Revista de Análisis Transaccional y Psicología Humanista, nº 60, p. 17-21.

Valbuena, F.(2007a). Análisis de la película Hotel Rwanda(2004), en Revista de Análisis Transaccional y Psicología Humanista, no 61, p. 71-79.

Valbuena, F.(2007b). Análisis de la película La vida de los otros (Desde el Análisis Transaccional y la Teoría de la Negociación), en Cuadernos de Información y Comunicación, no 12, p. 119-135.

Valbuena, F.(2009). El Guión de Vida del Dr. Gregory House, en Cuadernos de Información y Comunicación, nº 54, p. 159-198.

Valbuena, F. (2010). El humor en la Comunicación Política, en Cuadernos de Información y Comunicación, nº 15, p. 165-185.

\section{Recursos electrónicos:}

Padilla Castillo, G. (2010a). Las series de televisión sobre médicos (1990-2010): tres enfoques: comunicación interpersonal, comunicación institucional, relaciones entre ética, moral y política. Madrid: Universidad Complutense de Madrid. Consultado el 12 de Marzo del 2012. Disponible en: http://eprints.ucm.es/11399/1/T32391.pdf

Requeijo, P. (2012a). Estados del ego, transacciones, juegos y comunicación no verbal en "Luna Nueva", Tener y no Tener" y "Río Bravo" de Howard Hawks. Madrid: Universidad Complutense de Madrid. Consultado el 12 de Marzo del 
2012. Disponible en: http://eprints.ucm.es/16213/1/T33843.pdf

Rodríguez Vidales, Y. (2012). El Ala Oeste de la Casa Blanca y otros modelos de ficción para comprender la Comunicación Política. Madrid: Universidad Complutense de Madrid. . Consultado el 12 de Agosto del 2012. Disponible en: http://eprints.ucm.es/17551/1/T34094.pdf 